\title{
Vitamin D supplementation and COVID-19 risk: a population-based, cohort study
}

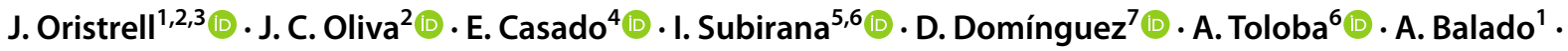 \\ M. Grau ${ }^{5,6,8}$ (1)
}

Received: 28 June 2021 / Accepted: 12 July 2021 / Published online: 17 July 2021

(C) Italian Society of Endocrinology (SIE) 2021

\begin{abstract}
Purpose To analyze the associations between cholecalciferol or calcifediol supplementation, serum 25-hydroxyvitamin D (25OHD) levels and COVID-19 outcomes in a large population.

Methods All individuals $\geq 18$ years old living in Barcelona-Central Catalonia ( $n=4.6$ million) supplemented with cholecalciferol or calcifediol from April 2019 to February 2020 were compared with propensity score-matched untreated controls. Outcome variables were SARS-CoV2 infection, severe COVID-19 and COVID-19 mortality occuring during the first wave of the pandemic. Demographical data, comorbidities, serum 25OHD levels and concomitant pharmacological treatments were collected as covariates. Associations between cholecalciferol or calcifediol use and outcome variables were analyzed using multivariate Cox proportional regression.

Results Cholecalciferol supplementation $(n=108,343)$ was associated with slight protection from SARS-CoV2 infection $(n=4352$ [4.0\%] vs 9142/216,686 [4.2\%] in controls; HR 0.95 [CI 95\% 0.91-0.98], $p=0.004)$. Patients on cholecalciferol treatment achieving $25 \mathrm{OHD}$ levels $\geq 30 \mathrm{ng} / \mathrm{ml}$ had lower risk of SARS-CoV2 infection, lower risk of severe COVID-19 and lower COVID-19 mortality than unsupplemented 25OHD-deficient patients (56/9474 [0.6\%] vs 96/7616 [1.3\%]; HR 0.66 [CI 95\% 0.46-0.93], $p=0.018)$. Calcifediol use $(n=134,703)$ was not associated with reduced risk of SARS-CoV2 infection or mortality in the whole cohort. However, patients on calcifediol treatment achieving serum 25OHD levels $\geq 30 \mathrm{ng} / \mathrm{ml}$ also had lower risk of SARS-CoV2 infection, lower risk of severe COVID-19, and lower COVID-19 mortality compared to 25OHD-deficient patients not receiving vitamin D supplements (88/16276 [0.5\%] vs 96/7616 [1.3\%]; HR 0.56 [CI 95\% $0.42-0.76], p<0.001$ ).
\end{abstract}

Conclusions In this large, population-based study, we observed that patients supplemented with cholecalciferol or calcifediol achieving serum $25 \mathrm{OHD}$ levels $\geq 30 \mathrm{ng} / \mathrm{ml}$ were associated with better COVID-19 outcomes.

Keywords Cholecalciferol · Calcifediol · Vitamin D · COVID-19 · SARS-CoV2

J. Oristrell

joristrell@tauli.cat

$\triangle$ E. Casado

ecasado@tauli.cat

1 Internal Medicine Service, Corporació Sanitària Parc Taulí, Parc Taulí s/n, 08208 Sabadell, Barcelona, Catalonia

2 Institut d'Investigació i Innovació I3PT, Sabadell, Catalonia

3 Department of Medicine, Universitat Autònoma de Barcelona, Barcelona, Catalonia

4 Rheumatology Service, Corporació Sanitària Parc Taulí, Parc Taulí s/n, 08208 Sabadell, Barcelona, Catalonia
5 CIBER of Epidemiology and Public Health (CIBERESP), Barcelona, Catalonia

6 Institut Hospital del Mar d'Investigacions Mèdiques, Barcelona, Catalonia

7 Agència de Qualitat i Avaluació Sanitària, Generalitat de Catalunya, Barcelona, Catalonia

8 Department of Medicine, University of Barcelona, Barcelona, Catalonia 


\section{Introduction}

Infection with the new coronavirus SARS-CoV2 is characterized by an important clinical variability, which suggests that there are important host-related factors that impact in COVID19 outcomes. One of these factors has been postulated to be vitamin $\mathrm{D}$ deficiency $[1,2]$, which is a prevalent condition worldwide [3]. Vitamin D is now being recognized as an hormonal system with many extra-skeletal actions, including important effects on the immunological system [4-6].

Several clinical trials and two meta-analysis have shown that cholecalciferol or ergocalciferol supplementation may help prevent acute respiratory infections [7, 8]. However, evidence that these drugs are helpful to treat or prevent SARSCoV2 infection is still controversial [9].

Observational studies have described an association between low serum levels of 25-hydroxyvitamin D (25OHD) and higher risk of SARS-CoV2 infection [10-12], higher risk of severe COVID-19 or higher COVID-19 mortality [13-15]. Three meta-analysis have also concluded that there is an association between low serum levels of $250 \mathrm{HD}$ and higher risk of COVID-19 mortality [16-18]. However, studies analyzing the use of cholecalciferol or calcifediol supplementation to modify COVID-19 outcomes have offered inconclusive results. While some observational studies in hospitalized patients have shown reduced COVID-19 severity or mortality in patients supplemented with cholecalciferol or calcifediol [19-21], Cereda et al. described a trend to an increased mortality in patients supplemented with calcifediol [22].

The effects of cholecalciferol or calcifediol as a treatment for hospitalized COVID-19 patients have also been studied in three low-powered clinical trials, without observing any significant reduction in COVID-19 mortality [23-25].

To the best of our knowledge, there are not published results describing the effects, at the population level, of cholecalciferol or calcifediol supplementation on COVID-19 outcomes. To ascertain whether vitamin D supplementation protects against SARS-CoV2 infection or from COVID-19 adverse outcomes, we designed this large observational study. We have already shown that calcitriol supplementation, the active form of vitamin D, mainly used in patients with advanced renal failure, was associated with important reductions in COVID-19 mortality [26]. Now, we present the results of cholecalciferol or calcifediol supplementation showing also beneficial effects of these drugs in patients that reach normal serum 25OHD levels.

\section{Methods}

\section{Study design and population included}

A retrospective cohort was built using the databases of the public healthcare system in Catalonia. We analyzed all individuals $\geq 18$ years old living in Barcelona and Central Catalonia regions on 25 February 2020, date of the first positive PCR for SARS-CoV2 in our country $(n=4,643,139)$.

In this population, we performed three independent studies to investigate the association of cholecalciferol or calcifediol supplementation with COVID-19 outcomes:

Comparison of COVID outcomes between supplemented patients and propensity score-matched controls: We identified all patients receiving cholecalciferol $(n=201,445)$ or calcifediol $(n=207,136)$ supplementation from 1 April 2019 to 28 February 2020 and patients not receiving any vitamin D supplement $(4,267,430)$ during the same period. Since chronic kidney disease (CKD) is a strong predictor of worse prognosis in COVID-19 [27], subjects without an available serum creatinine determination performed between 1 October 2018 and 28 February 2020 were excluded from the study. After propensity score matching (see below), 108,343 patients on cholecalciferol, 216,686 matched controls (cholecalciferol controls), 134,703 patients on calcifediol and 269,406 matched controls (calcifediol controls) were selected for the analysis. Association between mean daily cholecalciferol or calcifediol dose and COVID-19 outcomes: All patients receiving cholecalciferol or calcifediol supplementation from 1 November 2019 to 28 February 2020, with an available serum creatinine level $(n=165,588$ and 132,590 , respectively), were selected for this analysis. This shorter period of time was chosen to minimize the effects of eventual changes in the dose of these drugs. Comparison of COVID-19 outcomes between cholecalciferol-or calcifediol-supplemented patients with a sufficient vitamin D status (serum 25OHD> $30 \mathrm{ng}$ / $\mathrm{ml}$ ) and unsupplemented vitamin D-deficient (serum $25 \mathrm{OHD}<20 \mathrm{ng} / \mathrm{ml}$ ) patients: In order to reduce the variability in serum 250HD levels due to seasonal sun exposure, we only analyzed serum levels determined between 1 November 2019 and 28 February 2020. All patients of the cohort that had a serum $250 H D$ determination in this period of time $(n=85,158)$ were included in this analysis.

\section{Data sources}

Given Catalonia's universal health and medication coverage, we were able to utilize electronic databases to 
examine the association of cholecalciferol and calcifediol use with COVID-19 outcomes in a real world setting. We used anonymized data provided by the Catalan Agency for Health Quality and Evaluation (AQUAS) within the framework of the Data Analytics Program for Health Research and Innovation (PADRIS). PADRIS databases include information on demographics (age and sex), diagnoses, laboratory data, drugs supplied by pharmacies, Primary Care physician diagnoses, laboratory results and diagnoses, procedures and outcomes of medical admissions in the public hospitals in Catalonia. This project was approved in a public call for grants for using PADRIS databases in research projects on COVID-19.

\section{Identification of patients on cholecalciferol or calcifediol supplementation}

Patients who had been supplied in pharmacies with drugs of the Anatomical Therapeutic Chemical Classification System groups A11CC05, A12AX, M05BB03, M05BB07, M05BB08, M05BB09, A11CC06 or A11CC55 from 1 April 2019 to 28 February 2020 were analyzed. The sum of Defined Daily Doses (DDD) of cholecalciferol or the sum of calcifediol doses supplied from 1 November 2019 to 28 February 2020 were identified, transformed into micrograms, and the mean daily cholecalciferol or calcifediol dose received per patient, in micrograms, was calculated. Patients receiving formulations containing $>250 \mu \mathrm{g}$ of cholecalciferol (12.5 DDD) or $>250 \mu \mathrm{g}$ of calcifediol per dose were considered as receiving bolus doses.

\section{Identification of control subjects through propensity score matching}

We performed two independent propensity score matching to build the control groups for cholecalciferol and calcifediol using the 'Matching' package in R [28] as described [26]. First, we used multivariate logistic regression to model receiving or not each drug as a function of the following covariates: sex, age, fifteen comorbidities identified from the International Classification of Diseases (ICD-10) diagnostic codes issued by family physicians (Supplementary Table 1), estimated glomerular filtration rate (eGFR), history of cigarette smoking, nursing home residence and use of seven classes of drugs that could potentially affect the prognosis (Supplementary Table 1). Estimated glomerular filtration rate was obtained from serum levels of creatinine, sex and age according to the Chronic Kidney Disease Epidemiology Collaboration (CKD-EPI) equation [29]. Propensity scores were matched using the nearest-neighbor matching method without replacement at a 1:2 ratio of treated subjects and controls. A caliper of 0.2 of the standard deviation of the propensity score logit was established as the maximum tolerated difference between matched patients. To examine the balance of each covariate between the treatment and the control group, the standardized mean difference was calculated before and after matching using Tableone package in $\mathrm{R} \mathrm{[30].} \mathrm{We} \mathrm{considered} \mathrm{the} \mathrm{groups} \mathrm{well} \mathrm{balanced} \mathrm{if} \mathrm{the}$ standardized mean difference was $<0.10$ for each covariate.

\section{Serum levels of 25-hydroxyvitamin D}

Serum levels of 25OHD determined in the laboratories of the catalan public health system between 1 November 2019 and 28 February 2020 in the whole cohort were obtained from PADRIS databases. A deficient vitamin D status was defined as a serum $25 \mathrm{OHD}$ level $<20 \mathrm{ng} / \mathrm{mL}$ and a sufficient vitamin D status was defined as a serum $250 \mathrm{ODD}$ level $\geq 30 \mathrm{ng} / \mathrm{mL}$.

\section{Outcome variables}

We analyzed the occurence of SARS-CoV2 infection, COVID-19 hospitalization, intensive care admission, the procedures during hospitalization and mortality during the first wave of the pandemic. Four main outcome variables were defined, with different timings due to the natural course of the disease:

\section{SARS-CoV2 infection}

Positive PCR result for SARS-CoV2 or a clinical diagnosis made by a Primary Care physician, or a hospital discharge report stating a diagnosis of COVID-19 (ICD-10 codes used are displayed in Supplementary Table 1), from 25 February 2020 to 30 April 2020. Time (in days) from 24 February 2020 until a positive PCR or a clinical diagnosis (the first event) was used for survival analysis. Censored time for those individuals without the event was the time from 24 February to 30 April 2020.

\section{COVID-19 mortality}

Patients diagnosed with COVID-19 infection resulting in death between 25 February and 15 May 2020. Patients with COVID-19 admitted to hospital before 16 May 2020 and dying before 7 June were also included. Time (in days) from 24 February 2020 to COVID-19 death was used for survival analysis. Censored time for those individuals without the event was the time from 24 February to 7 June 2020.

\section{Severe COVID-19}

Composite outcome of COVID-19 mortality, as already defined, or COVID-19 hospital admission needing non-invasive mechanical ventilation, orotracheal intubation, mechanical ventilation or intensive care unit admission from 25 February 
2020 to 15 May 2020. Time (in days) from 24 February 2020 until hospital admission (if severe COVID-19 developed during hospitalization) or time (in days) from 24 February 2020 until COVID-19 death was used for survival analysis. Censored time for those individuals without the event was the time from 24 February to 7 June 2020.

\section{Statistical analysis}

Continous variables are reported as mean and standard deviation and qualitative variables are summarized by frequencies and percentages. Basal differences between treated and untreated groups were assessed using Student's t test or chisquare test and standardized mean differences.

Once the control groups were established, associations between cholecalciferol or calcifediol supplementation and outcome variables were further analyzed using unadjusted and multivariate Cox proportional hazards regression models. All the variables that approached statistical significance $(p<0.2)$ were initially selected for inclusion in the adjusted analyses. Multivariable models were constructed by means of a stepwise forward inclusion procedure and only the significant variables were retained in the final model. Unadjusted and adjusted hazard ratios and their 95\% confidence intervals are reported.

Taking into account that vitamin D supplementation may be prescribed to treat a low vitamin D status, we also compared the outcome variables between patients with sufficient vitamin D status, while being vitamin D-supplemented, with patients deficient in vitamin $\mathrm{D}$ and not supplemented, also using multivariate Cox regression analysis. Finally, the associations between the mean daily cholecalciferol or mean daily calcifediol dose and COVID-19 outcomes were also analyzed using multivariate Cox regression analysis.

For all statistical tests, a $p$ value $<0.05$ was used for statistical significance.

Descriptive statistics and survival analysis were carried out using SPSS version 25.0 for Windows (SPSS, Chicago, IL, USA), and Survival and Survminer packages in R [31, 32].

\section{Ethical issues and confidenciality}

All data were treated anonymously in order for this study to comply with the provisions of Spanish and European laws on Protection of Personal Data. The study was approved by the ethics committee of the Corporació Sanitària Parc TaulíUniversitat Autònoma de Barcelona.

\section{Results}

\section{COVID-19 outcomes in cholecalciferol-supplemented patients versus propensity score-matched controls}

A total of 201,445 patients $\geq 18$ years old were identified as being on cholecalciferol treatment between 1 April 2019 and 28 February 2020 in Barcelona and Central Catalonia regions. After propensity-score matching, 108,343 patients on cholecalciferol and 216,686 matched control patients were included in the analysis.

Clinical characteristics of the patients treated with cholecalciferol and their matched controls are shown in Table 1. Mean age was 70 years and more than $83 \%$ were women. SARS-CoV2 infection was diagnosed in 4352 patients supplemented with cholecalciferol (2113 of them [48.6\%] confirmed by PCR) and in 9142 untreated controls (4300 of them [47.0\%] confirmed by PCR). Cholecalciferol use was associated with a mild, but significant, lower risk of SARS-CoV2 infection, both in the univariate and in the multivariate Cox regression analysis $(n=4352$ [4.0\%] versus $n=9142$ [4.2\%] in controls; HR 0.95 [CI 95\% 0.91-0.98], $p=0.004)$. This mild reduction in the infection rate was not observed in the subgroup of patients diagnosed by PCR (HR 0.98 [0.93-1.04; $p=0.51]$. We did not observe any significant association between cholecalciferol supplementation and COVID-19 severity $(n=798$ [0.7\%] versus $n=1650$ [0.8\%] in controls) or COVID-19 mortality $(\mathrm{n}=716[0.7 \%]$ versus $n=1492[0.7 \%]$ in controls), when comparing with matched untreated controls (Table 2).

Patients receiving bolus cholecalciferol had lower COVID-19 mortality (100/20,715; [0.5\%]) than patients on daily cholecalciferol treatment $(616 / 87628 ;[0.7 \%])$. However, in the multivariate Cox regression analysis, receiving bolus cholecalciferol was not significantly associated with lower COVID-19 severity nor mortality.

We analyzed the cholecalciferol DDD received in the 165,588 patients that had been supplied between 1 November 2019 and 28 February 2020. Mean daily cholecalciferol doses were similar in patients who became infected or died due to COVID-19 than in patients without these outcomes (Table 3). However, in the multivariate Cox regression analysis, the mean daily dose of cholecalciferol, measured in $10 \mu \mathrm{g}$ intervals (equivalent to $400 \mathrm{IU}$ ), was associated with small but significant reductions in the risk of SARSCov2 infection (HR 0.97 [CI 95\% 0.95-0.99], $p=0.007$ ), in SARS-CoV2 infection confirmed by PCR (HR 0.94 [CI 95\% 0.91-0.97], $p<0.001$ ), in severe COVID-19 (HR 0.92 [CI 95\% 0.87-0.97], $p=0.002$ ) and in COVID-19 mortality (HR 0.90 [CI 95\% 0.85-0.96], $p=0.001$ ) (Table 3). 
Table 1 Clinical characteristics of patients on cholecalciferol or calcifediol treatment and their matched controls

\begin{tabular}{|c|c|c|c|c|c|c|}
\hline & $\begin{array}{l}\text { Cholecalciferol } \\
\text { treated }(n=108,343)\end{array}$ & $\begin{array}{l}\text { Cholecalcif- } \\
\text { erol matched } \\
(n=216,686)\end{array}$ & SMD & $\begin{array}{l}\text { Calcifediol treated } \\
(n=134,703)\end{array}$ & $\begin{array}{l}\text { Calcife- } \\
\text { diol matched } \\
(n=269,406)\end{array}$ & SMD \\
\hline \multicolumn{7}{|l|}{ Variables used for matching } \\
\hline Female gender, $n(\%)$ & $90,417(83.5)$ & $181,414(83.7)$ & 0.007 & $105,229(78.1)$ & 209,824 (77.9) & 0.006 \\
\hline Age, mean (SD) & $70.0(14.0)$ & $70.0(14.6)$ & 0.001 & $68.8(14.9)$ & $68.8(15.1)$ & 0.004 \\
\hline Nursing home residence, $n(\%)$ & $2884(2.7)$ & $5649(2.6)$ & 0.003 & $3548(2.6)$ & $6989(2.6)$ & 0.002 \\
\hline Cigarette smoking, $n(\%)$ & $23,329(21.5)$ & $46,003(21.2)$ & 0.007 & $32,507(24.1)$ & $64,571(24.0)$ & 0.004 \\
\hline \multicolumn{7}{|l|}{ Comorbidities } \\
\hline Hypertension, $n(\%)$ & $59,157(54.6)$ & $116,565(53.8)$ & 0.016 & $75,058(55.7)$ & $148,784(55.2)$ & 0.010 \\
\hline Obesity, $n(\%)$ & $39,941(36.9)$ & $78,711(36.3)$ & 0.011 & $51,547(38.3)$ & $102,367(38.0)$ & 0.006 \\
\hline Diabetes, $n(\%)$ & $23,899(22.1)$ & $46,978(21.7)$ & 0.009 & $33,284(24.7)$ & $66,443(24.7)$ & 0.001 \\
\hline Heart failure, $n(\%)$ & $11,572(10.7)$ & $22,939(10.6)$ & 0.003 & $14,692(10.9)$ & $29,260(10.9)$ & 0.001 \\
\hline $\mathrm{COPD}, n(\%)$ & $14,838(13.7)$ & $29,376(13.6)$ & 0.004 & $18,431(13.7)$ & $36,947(13.7)$ & 0.001 \\
\hline Asthma, $n(\%)$ & $11,791(10.9)$ & $23,332(10.8)$ & 0.004 & $13,983(10.4)$ & $27,894(10.4)$ & 0.001 \\
\hline eGFR, mean (SD) & $78.35(20.65)$ & $78.46(20.96)$ & 0.005 & $78.01(22.91)$ & $78.01(21.62)$ & $<0.001$ \\
\hline Cerebrovascular disease, $n(\%)$ & 7949 (7.3) & $15,674(7.2)$ & 0.004 & $10,234(7.6)$ & $20,346(7.6)$ & 0.002 \\
\hline Dementia, $n(\%)$ & $6517(6.0)$ & $12,783(5.9)$ & 0.005 & $7841(5.8)$ & $15,590(5.8)$ & 0.001 \\
\hline Malignant neoplasia, $n(\%)$ & $28,731(26.5)$ & $57,158(26.4)$ & 0.003 & $32,604(24.2)$ & $65,240(24.2)$ & $<0.001$ \\
\hline Liver cirrhosis, $n(\%)$ & $1426(1.3)$ & $2846(1.3)$ & $<0.001$ & $1641(1.2)$ & $3346(1.2)$ & 0.002 \\
\hline Osteoporosis, $n(\%)$ & $19,332(17.8)$ & $37,668(17.4)$ & 0.012 & $19,052(14.1)$ & $36,908(13.7)$ & 0.013 \\
\hline Past femur fracture, $n(\%)$ & $1746(1.6)$ & $2939(1.4)$ & 0.021 & $1676(1.2)$ & $2877(1.1)$ & 0.016 \\
\hline Dyslipidemia, $n(\%)$ & $57,688(53.2)$ & $115,410(53.3)$ & $<0.001$ & $70,817(52.6)$ & $142,020(52.7)$ & 0.003 \\
\hline Ischemic heart disease, $n(\%)$ & $7204(6.6)$ & $14,097(6.5)$ & 0.006 & $10,331(7.7)$ & $20,784(7.7)$ & 0.002 \\
\hline Peripheral arteriopathy, $n(\%)$ & $3085(2.8)$ & $6064(2.8)$ & 0.003 & 4679 ( 3.5$)$ & $9372(3.5)$ & $<0.001$ \\
\hline \multicolumn{7}{|l|}{ Use of drugs } \\
\hline Proton pump inhibitors & $49,488(45.7)$ & $98,961(45.7)$ & $<0.001$ & $58,522(43.4)$ & $118,032(43.8)$ & 0.007 \\
\hline Oral corticosteroids, $n(\%)$ & $11,986(11.1)$ & $24,149(11.1)$ & 0.003 & $11,098(8.2)$ & $22,562(8.4)$ & 0.005 \\
\hline DPP4-inhibitors, $n(\%)$ & $2659(2.5)$ & $5194(2.4)$ & 0.004 & $4220(3.1)$ & $8440(3.1)$ & $<0.001$ \\
\hline Statins, $n(\%)$ & $33,471(30.9)$ & $66,992(30.9)$ & $<0.001$ & $43,130(32.0)$ & $86,714(32.2)$ & 0.004 \\
\hline ACE inhibitors, $n(\%)$ & $26,353(24.3)$ & $52,413(24.2)$ & 0.003 & $32,101(23.8)$ & $64,087(23.8)$ & 0.001 \\
\hline $\mathrm{ARB}, n(\%)$ & $18,988(17.5)$ & $37,532(17.3)$ & 0.005 & 25,486 (18.9) & $50,685(18.8)$ & 0.003 \\
\hline Immunosuppressants, $n(\%)$ & 3095 (2.9) & $6082(2.8)$ & 0.003 & $3004(2.2)$ & $5941(2.2)$ & 0.002 \\
\hline
\end{tabular}

$S M D$ standardized mean difference, $S D$ standard deviation, $C O P D$ chronic obstructive pulmonary disease, $e G F R$ estimated glomerular filtration rate, $A C E$ angiotensin convertint enzyme, $A R B$ angiotensin-II receptor blockers, $D P P 4$ dipeptidyl peptidase-4

\section{COVID-19 outcomes in calcifediol-supplemented patients versus propensity score-matched controls}

A total of 207,136 patients $\geq 18$ years old were identified as being on calcifediol treatment between 1 April 2019 and 28 February 2020 in Barcelona-Central Catalonia region. After propensity-score matching, 134,703 patients on calcifediol and 269,406 matched control patients were included in the study. Calcifediol was supplied as bolus doses in $99.4 \%$ of patients or as a daily drop formulation in $0.6 \%$ of patients.

Clinical variables in patients treated with calcifediol and their respective control group are shown in Table 1. Mean age of patients was 69 years, with a high proportion of women and similar comorbidities to the patients treated with cholecalciferol.
SARS-CoV2 infection was diagnosed in 5662 patients supplemented with calcifediol (2607 of them [46.0\%] confirmed by PCR) and in 11,401 untreated controls (5413 of them [47.5\%] confirmed by PCR). We did not observe any significant association between calcifediol supplementation and the risk of SARS-CoV2 infection $(n=5662$ [4.2\%] versus 11,401 [4.2\%] in matched controls), nor between calcifediol use and COVID-19 severity $(n=1037$ [0.8\%] versus $n=2073[0.8 \%]$ in controls) or mortality $(n=934$ [0.7\%] versus $n=1859[0.7 \%]$ in controls) (Table 4).

Among calcifediol treated patients, 132,590 had received the drug between 1 November 2019 and 28 February 2020. In the multivariate Cox regression analysis, the mean daily calcifediol dose received in this period, measured in $10 \mu \mathrm{g}$ intervals, was not associated with the risk of SARS-CoV2 


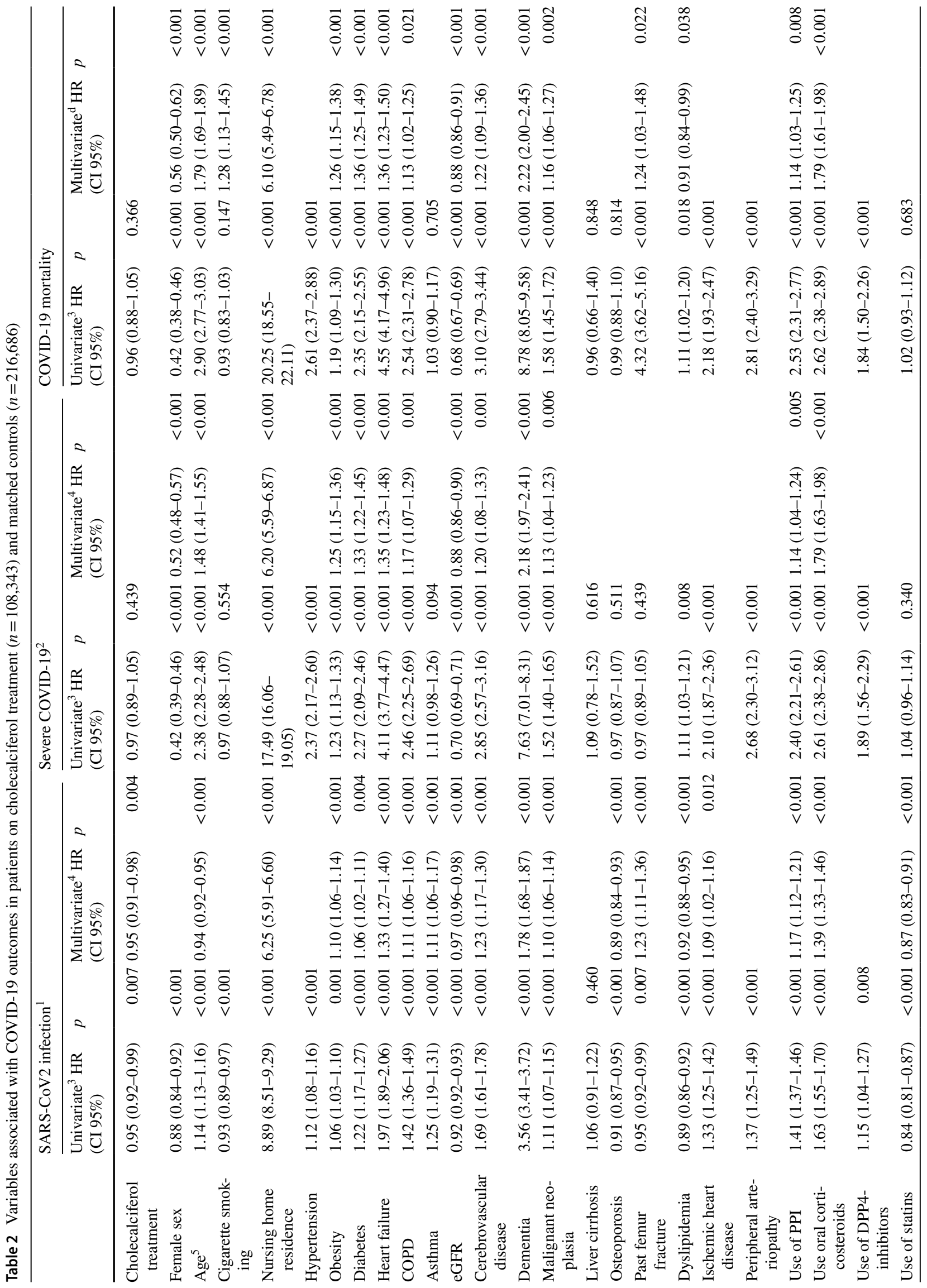


infection, nor with COVID-19 severity or mortality (Table 3).

Since renal proximal tubular reabsortion of calcifediol may be impaired in CKD, and low serum calcifediol levels are a frequent finding in this disease [33], we also analyzed the association between calcifediol supplementation and COVID-19 outcomes in the subgroup of patients with advanced CKD (stages 4 and 5). In these patients, calcifediol supplementation was associated with a significant reduction in SARS-CoV2 infection (311/4380 [7.1\%] vs 568/5533 [10.3\%] in untreated controls; HR 0.77 [0.67-0.88]; $p<0.001$ in multivariate Cox proportional hazards regression analysis) and a reduced risk of severe COVID-19 (144/4380; [3.3\%] vs 260/5533 [4.7\%] in untreated controls; HR 0.78 [0.63-0.96]; $p<0.001$ ). A reduction in COVID-19 mortality was also observed in patients with advanced CKD supplemented with calcifediol, but without reaching statistical significance (138/4380; [3.2\%] vs 245/5533; [4.4\%]; HR 0.83 [0.67-1.02]; $p=0.077)$. These associations were not observed in patients with better renal function (Table 5).

\section{Serum 25-hydroxyvitamin D levels and COVID-19 risk.}

Serum 25OHD levels were determined in 85,158 patients in the whole cohort $(71,972$ patients on cholecalciferol or calcifediol supplementation and 13,186 patients of the untreated control groups) between 1 November 2019 and 28 February 2020.

Mean serum 25OHD levels were significantly lower in patients that developed SARS-CoV2 infection (22.7 [SD $14.1] \mathrm{ng} / \mathrm{ml}, n=3091)$ than in non-infected patients (24.0 [14.1] ng/ml, $p<0.001)$. Mean serum 25OHD levels were also significantly lower in patients that developed severe COVID-19 (22.0 [SD 15.7] ng/ml $[n=538]$ versus 24.0 [14.3] $\mathrm{ng} / \mathrm{ml}, p=0.004)$ and in patients that died due to COVID (21.9 [15.7] ng/ml [ $n=475]$ versus 24.0 [14.3] ng/ $\mathrm{ml}, p=0.004)$.

In the multivariate analysis, lower serum 25OHD levels were also associated with increased risk of SARS-CoV2 infection (HR 0.97 [CI 95\% 0.94-0.99]; $p=0.017$ ), but were not significantly associated with severe COVID-19 or with COVID-19 mortality.

\section{COVID-19 outcomes}

in cholecalciferol-supplemented, vitamin D-sufficient patients, versus unsupplemented, vitamin D-deficient patients.

The rate of SARS-CoV2 infection was significantly lower in vitamin D-sufficient patients supplemented with cholecalciferol (309/9474 [3.3\%]) than in patients vitamin D deficient not receiving vitamin D supplements (430/7616 [5.6\%]; 


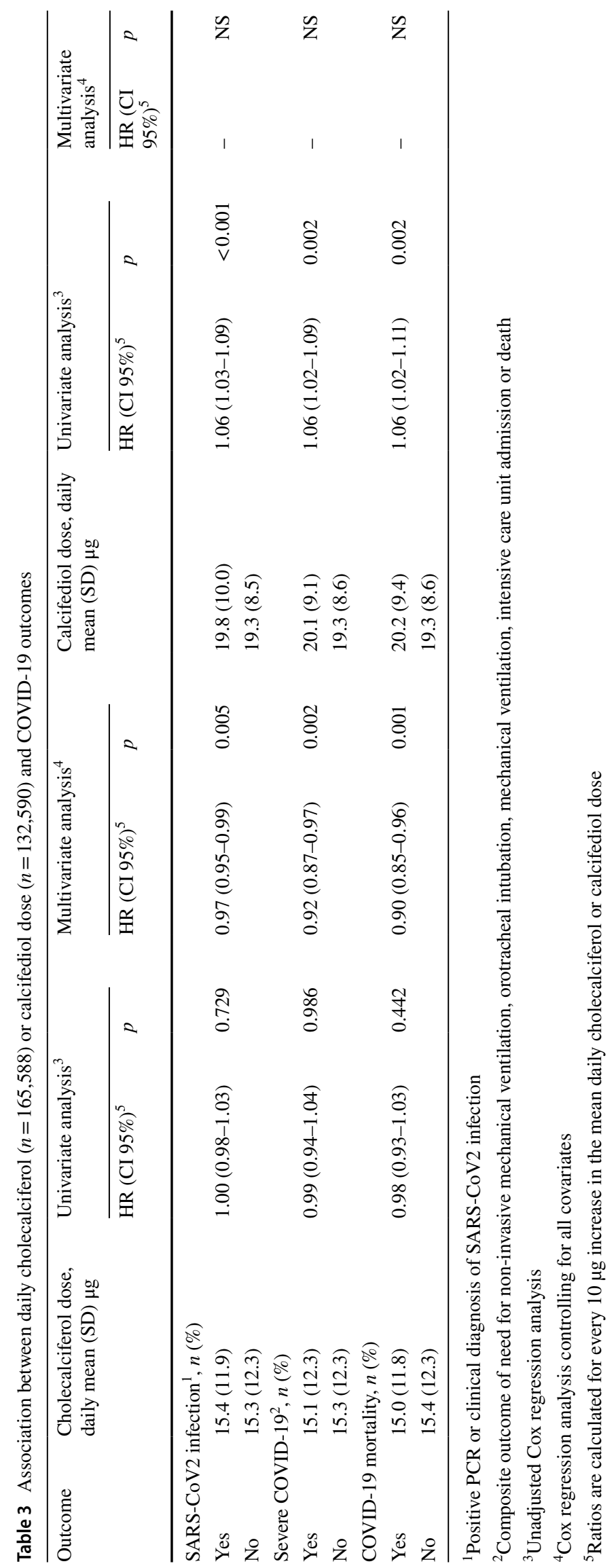


Table 4 SARS-CoV2 infection, severe infection and death in patients treated with calcifediol $(n=134,703)$ and their matched controls $(269,406)$

\begin{tabular}{|c|c|c|c|c|c|c|}
\hline \multirow[t]{2}{*}{ Outcome } & \multirow{2}{*}{$\begin{array}{l}\text { Calcifediol treatment } \\
(n=134,703)\end{array}$} & \multirow{2}{*}{$\begin{array}{l}\text { Matched controls } \\
(n=269,406)\end{array}$} & \multicolumn{2}{|c|}{ Univariate analysis $^{3}$} & \multicolumn{2}{|c|}{ Multivariate analysis ${ }^{4}$} \\
\hline & & & HR (CI 95\%) & $p$ & HR (CI 95\%) & $p$ \\
\hline SARS-CoV2 infection ${ }^{1}, n(\%)$ & $5662(4.2)$ & $11,401(4.2)$ & $0.99(0.96-1.03)$ & 0.646 & - & - \\
\hline Severe COVID- $19^{2}, n(\%)$ & $1037(0.8)$ & $2073(0.8)$ & $1.00(0.93-1.08)$ & 0.995 & - & - \\
\hline COVID-19 mortality, $n(\%)$ & $934(0.7)$ & $1859(0.7)$ & $1.01(0.93-1.09)$ & 0.908 & - & - \\
\hline
\end{tabular}

${ }^{1}$ Positive PCR or clinical diagnosis of SARS-CoV2 infection

${ }^{2}$ Composite outcome of need for non-invasive mechanical ventilation, orotracheal intubation, mechanical ventilation, intensive care unit admission or death

${ }^{3}$ Unadjusted Cox regression analysis

${ }^{4}$ Cox regression analysis controlling for all covariates

Table 5 SARS-CoV2 infection, severe infection and death in patients treated with calcifediol and controls, according to CKD stages

\begin{tabular}{|c|c|c|c|c|c|c|}
\hline \multirow[t]{2}{*}{ Patients on stages $1-3 \mathrm{CKD}$} & \multirow{2}{*}{$\begin{array}{l}\text { Calcifediol } \\
(n=130,323)\end{array}$} & \multirow[t]{2}{*}{ Controls $(n=263,873)$} & \multicolumn{2}{|c|}{ Univariate analysis $^{3}$} & \multicolumn{2}{|c|}{ Multivariate analysis ${ }^{4}$} \\
\hline & & & HR (CI 95\%) & $p$ & HR (CI 95\%) & $p$ \\
\hline SARS-CoV2 infection ${ }^{1}, n(\%)$ & $5351(4.1)$ & $10,833(4.1)$ & $1.00(0.97-1.03)$ & 0.96 & - & NS \\
\hline Severe COVID- $19^{2}, n(\%)$ & $893(0.7)$ & $1813(0.7)$ & $1.00(0.92-1.08)$ & 0.94 & - & NS \\
\hline COVID-19 mortality, $n(\%)$ & $796(0.6)$ & $1614(0.6)$ & $1.00(0.92-1.09)$ & 0.97 & - & NS \\
\hline Patients on stages 4-5 CKD & $\begin{array}{l}\text { Calcifediol } \\
(n=4380)\end{array}$ & Controls $(n=5533)$ & HR (CI 95\%) & $p$ & HR (CI 95\%) & $p$ \\
\hline 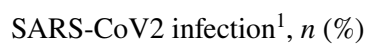 & $311(7.1)$ & $568(10.3)$ & $0.68(0.59-0.78)$ & $<0.001$ & $0.77(0.67-0.88)$ & $<0.001$ \\
\hline Severe COVID- $19^{2}, n(\%)$ & $144(3.3)$ & $260(4.7)$ & $0.69(0.57-0.85)$ & $<0.001$ & $0.78(0.63-0.96)$ & 0.018 \\
\hline COVID-19 mortality, $n(\%)$ & $138(3.2)$ & $245(4.4)$ & $0.71(0.57-0.87)$ & 0.001 & $0.83(0.67-1.02)$ & 0.077 \\
\hline
\end{tabular}

CKD chronic kidney disease

${ }^{1}$ Positive PCR or clinical diagnosis of SARS-CoV2 infection

${ }^{2}$ Composite outcome of need for non-invasive mechanical ventilation, orotracheal intubation, mechanical ventilation, intensive care unit admission or death

${ }^{3}$ Unadjusted Cox regression analysis

${ }^{4}$ Cox regression analysis controlling for all covariates

HR 0.66 [CI 95\% 0.57-0.77], $p<0.001$ in multivariate Cox proportional hazards regression analysis). Similarly, vitamin D-sufficient patients on cholecalciferol supplementation had lower risk of severe COVID-19 (65/9474 [0.7\%] vs 99/7616 [1.3\%]; HR 0.72 [0.52-1.00]; $p=0.050)$, and lower COVID19 mortality (56/9474 [0.6\%] vs 96/7616 [1.3\%]; HR 0.66 [CI 95\% 0.46-0.93], $p=0.018$ ) compared to vitamin D-deficient unsupplemented patients (Table 6).

\section{COVID-19 outcomes in calcifediol-supplemented, vitamin D-sufficient patients, versus unsupplemented, vitamin D-deficient patients.}

The rate of SARS-CoV2 infection was significantly lower in vitamin D-sufficient patients supplemented with calcifediol (535/16276 [3.3\%]) than in patients vitamin D deficient not receiving vitamin D supplements (430/7616 [5.6\%]; HR 0.69
[CI 95\% 0.61-0.79], $p<0.001$ in multivariate Cox proportional hazards regression analysis). Similarly, vitamin D-sufficient patients on calcifediol supplementation had lower risk of severe COVID-19 $(n=100 / 16276$ [0.6\%] vs $n=99 / 7616$ [1.3\%]; HR 0.61 [0.46-0.81]; $p=0.001$ ), and lower COVID19 mortality $(n=88 / 16276$ [0.5\%] vs $n=96 / 7616$ [1.3\%]; HR 0.56 [0.42-0.76]; $p<0.001)$ compared to vitamin D-deficient unsupplemented patients (Table 7).

\section{Discussion}

In this large population-based cohort, we have compared COVID-19 outcomes in patients supplemented with cholecalciferol or calcifediol versus untreated matched controls, finding only a mild reduction in the risk of SARS-CoV2 infection (diagnosed clinically or by PCR) in patients supplemented with cholecalciferol, and a small reduction in 
Table 6 COVID-19 outcomes in 25OHD-sufficient, cholecalciferol-supplemented patients compared to unsupplemented, 25OHD-deficient patients

\begin{tabular}{|c|c|c|c|c|c|c|}
\hline & \multirow{2}{*}{$\begin{array}{l}\text { Cholecalciferol treated and } \\
\text { serum 25OHD } \geq 30 \mathrm{ng} / \mathrm{ml} \\
(n=9474)\end{array}$} & \multirow{2}{*}{$\begin{array}{l}\text { Untreated controls with } \\
\text { serum } 25 \mathrm{OHD}<20 \mathrm{ng} / \mathrm{ml} \\
(n=7616)\end{array}$} & \multicolumn{2}{|c|}{ Univariate analysis $^{3}$} & \multicolumn{2}{|c|}{ Multivariate analysis ${ }^{4}$} \\
\hline & & & HR (CI 95\%) & $p$ & HR (CI 95\%) & $p$ \\
\hline $\begin{array}{l}\text { SARS-CoV2 infection }{ }^{1}, \\
n(\%)\end{array}$ & $309(3.3 \%)$ & $430(5.6 \%)$ & $0.57(0.50-0.66)$ & $<0.001$ & $0.66(0.57-0.77)$ & $<0.001$ \\
\hline Severe COVID- $19^{2}, n(\%)$ & $65(0.7 \%)$ & $99(1.3 \%)$ & $0.53(0.39-0.72)$ & $<0.001$ & $0.72(0.52-1.00)$ & 0.050 \\
\hline COVID-19 mortality, $n(\%)$ & $56(0.6 \%)$ & $96(1.3 \%)$ & $0.47(0.34-0.65)$ & $<0.001$ & $0.66(0.46-0.93)$ & 0.018 \\
\hline
\end{tabular}

25OHD 25-hydroxyvitamin D

${ }^{1}$ Positive PCR or clinical diagnosis of SARS-CoV2 infection

${ }^{2}$ Composite outcome of need for non-invasive mechanical ventilation, orotracheal intubation, mechanical ventilation, intensive care unit admission or death

${ }^{3}$ Unadjusted Cox regression analysis

${ }^{4}$ Cox regression analysis controlling for all covariates

Table 7 COVID-19 outcomes in 25OHD-suficient, calcifediol-supplemented patients compared to unsupplemented, 25OHD-suficient patients

\begin{tabular}{|c|c|c|c|c|c|c|}
\hline & \multirow{2}{*}{$\begin{array}{l}\text { Calcifediol treated and } \\
\text { serum } 25 \mathrm{OHD} \geq 30 \mathrm{ng} / \mathrm{ml} \\
(n=16,276)\end{array}$} & \multirow{2}{*}{$\begin{array}{l}\text { Untreated controls with } \\
\text { serum } 25 \mathrm{OHD}<20 \mathrm{ng} / \mathrm{ml} \\
(n=7616)\end{array}$} & \multicolumn{2}{|c|}{ Univariate analysis $^{3}$} & \multicolumn{2}{|c|}{ Multivariate analysis ${ }^{4}$} \\
\hline & & & HR (CI 95\%) & $p$ & HR (CI 95\%) & $p$ \\
\hline $\begin{array}{l}\text { SARS-CoV2 infection }{ }^{1}, \\
n(\%)\end{array}$ & $535(3.3 \%)$ & $430(5.6 \%)$ & $0.58(0.51-0.66)$ & $<0.001$ & $0.69(0.61-0.79)$ & $<0.001$ \\
\hline Severe COVID- $19^{2}, n(\%)$ & $100(0.6 \%)$ & $99(1.3 \%)$ & $0.47(0.36-0.62)$ & $<0.001$ & $0.61(0.46-0.81)$ & 0.001 \\
\hline COVID-19 mortality, $n(\%)$ & $88(0.5 \%)$ & $96(1.3 \%)$ & $0.43(0.32-0.57)$ & $<0.001$ & $0.56(0.42-0.76)$ & $<0.001$ \\
\hline
\end{tabular}

25OHD 25-hydroxyvitamin D

${ }^{1}$ Positive PCR or clinical diagnosis of SARS-CoV2 infection

${ }^{2}$ Composite outcome of need for non-invasive mechanical ventilation, orotracheal intubation, mechanical ventilation, intensive care unit admission or death

${ }^{3}$ Unadjusted Cox regression analysis

${ }^{4}$ Cox regression analysis controlling for all covariates

the rates of infection and COVID-19 mortality associated with the use of higher cholecalciferol doses. Since vitamin D supplementation may be prescribed as a result of a low vitamin D status, we also compared COVID-19 outcomes between supplemented, vitamin D-sufficient patients with unsupplemented, vitamin D-deficient patients showing a significant reduction in the risk of infection, hospitalization and COVID-19 mortality in patients with a normal vitamin D status supplemented either with cholecalciferol or calcifediol. Overall, our results suggest that reaching a sufficient vitamin D status in patients supplemented with these vitamin D metabolites is associated with a reduced risk of SARS-CoV2 infection and lower COVID-19 mortality.

There are several pathophysiological mechanisms that could explain the benefits of vitamin D against COVID19. Calcitriol, the hormonal form of vitamin $\mathrm{D}$, can protect against infections by increasing the production of LL-37, $\beta$-defensin 2 and nitric oxide in respiratory epithelia [34] and it has been shown to reduce the incidence of adult respiratory distress syndrome in experimental models of lipopolysaccharide-induced acute lung injury [35-37].

From the methodological point of view, our study differs from previously published studies, where the effects of vitamin D supplementation were mostly analyzed in hospitalized COVID-19 patients [20-25]. To the best of our knowledge, this is the largest study to analyze the association of cholecalciferol or calcifediol supplementation with COVID-19 outcomes at the population level. This has allowed to detect small differences in outcomes after adjusting for multiple covariates. Our study also differs from that of Loucera et al., a study that compares COVID-19 inhospital mortality in patients under vitamin D supplementation with unsupplemented, propensity score-matched, hospitalized COVID-19 patients, since it only analyzed hospitalized patients and not all the population at risk [38].

Our study has some similarities with that of Meltzer et al. [11], who also combined the results of serum 25OHD levels and the vitamin D supplied to categorize 489 patients from an urban academic center as likely vitamin D sufficient or 
likely deficient; however, in that study, the authors only analyzed the risk of SARS-CoV2 infection, but not the risk of severe COVID-19 or COVID-19 mortality.

We also found a lower risk of SARS-CoV2 infection and reduced COVID-19 mortality in patients in stages 4-5 CKD supplemented with calcifediol. These results are similar, but of lower magnitude, than those observed in patients treated with calcitriol [26]. The better results obtained with calcitriol may be a consequence of its greater potency, being the active metabolite of vitamin D, not requiring any hydroxylation process in cytochromes and having a high affinity for the vitamin D receptor [39]. However, since renal proximal tubular cells express both ACE2 and mitochondrial 1-hydroxylase [40, 41], and they are commonly infected and damaged in severe COVID-19 [42, 43], it is also possible that during SARS-CoV2 infection, an acute decrease in renal calcitriol synthesis takes place that cannot be restored through cholecalciferol or calcifediol supplementation.

We think our study has some strengths, including the assessment of COVID-19 outcomes in a large population under supplementation with cholecalciferol or calcifediol and the use of a matched cohort of controls. This study also has some limitations. First, there are the inherent limitations of an observational cohort. Although we were comprehensive in analyzing many covariables, it is possible that there are still important variables not considered in the matching process that may disbalance the treated and control groups. Second, our data were obtained from the registries of the health administration of the government of Catalonia, which are fed by the diagnoses issued by family physicians, hospital discharge reports, laboratory data of public hospitals, or medicines supplied by pharmacies, with the inherent limitations of administrative data. Finally, we decided to focus our analysis on the first wave of the pandemic, with higher number of severe cases and mortality. However, the diagnosis of SARS-CoV2 in that phase could not be ascertained with PCR in all the cases, and some patients received a clinical diagnosis without a confirmatory microbiological confirmation.

In conclusion, in this large, population-based study, we observed that cholecalciferol or calcifediol supplementation seem to be beneficial against SARS-CoV2 infection, COVID-19 severity and COVID-19 mortality in patients achieving serum $25 \mathrm{OHD}$ levels $\geq 30 \mathrm{ng} / \mathrm{ml}$.

Supplementary Information The online version contains supplementary material available at https://doi.org/10.1007/s40618-021-01639-9.

Acknowledgements Agència de Qualitat i Avaluació Sanitàries (AQUAS). Programa d'Analítica de Dades per a la Recerca i la Innovació en Salut (PADRIS). Generalitat de Catalunya.

Author contributions JO conceptualization, data analysis, writing original draft. JCO design and methodological support. Formal data analysis. IS methodological support in the matching process. DD data curation. EC design, writing review. AT methodological support in the matching process. AB conceptualization and design. MG design and methodological support, writing review.

Funding This research received no external funding. The Catalan Agency for Health Quality and Evaluation (AQUAS) offered a data analyst for extracting all the data necessary for the development of this project.

Data availability statement All data can be offered to researchers upon request.

\section{Declarations}

Conflicts of interest The authors declare no conflict of interest. The Catalan Agency for Health Quality and Evaluation (AQUAS), a governmental office, offered a data analyst for extracting all the data necessary for the development of this project. They had no role in the design of the study, analyses, interpretation of data; in the writing of the manuscript, or in the decision to publish these results.

Institutional review board statement This study was approved by the Institutional Ethics Committee of the Corporació Sanitària Parc Taulí-Universitat Autònoma Barcelona (Project number 2020588).

Informed consent Not applicable.

Patient and public involvement It was not appropriate or possible to involve patients or the public in the design, or conduct, or reporting, or dissemination plans of our research.

Transparency statement The lead author (J. Oristrell) affirms that the manuscript is an honest, accurate, and transparent account of the study being reported. No important aspects of the study have been omitted, and there were no significant discrepancies from the study as it was planned.

Dissemination declaration Dissemination to the patients supplemented with vitamin D compounts is not applicable.

\section{References}

1. Grant WB, Lahore H, McDonnell SL, Baggerly CA, French CB, Aliano JL et al (2020) Evidence that vitamin D supplementation could reduce risk of influenza and COVID-19 infections and deaths. Nutrients 12:988. https://doi.org/10.3390/nu12040988

2. Teymoori-Rad M, Marashi SM (2020) Vitamin D and COVID19: from potential therapeutic effects to unanswered qüestions. Rev Med Virol. https://doi.org/10.1002/rmv.2159

3. Hilger J, Friedel A, Herr R, Rausch T, Roos F, Wahl DA et al (2014) A systematic review of vitamin D status in populations worldwide. Br J Nutr 111:23-45. https://doi.org/10.1017/S0007 114513001840

4. Peelen E, Knippenberg S, Muris AH, Thewissen M, Smolders J, Cohen Tervaert JW et al (2011) Effects of vitamin D on the peripheral adaptive immune system: a review. Autoimmun Rev 10:733-743

5. Kongsbak M, Levring TB, Geisler C, von Essen MR (2013) The vitamin $\mathrm{D}$ receptor an $\mathrm{T}$ cell function. Front Immunol 4:1-10 
6. Bscheider M, Butcher EC (2016) Vitamin D immunoregulation through dendritic cells. Immunology 148:227-236. https://doi. org/10.1111/imm.12610

7. Bergman P, Lindh AU, Björkhem-Bergman L et al (2013) Vitamin D and respiratory tract infections: a systematic review and meta-analysis of randomized controlled trials. PLoS ONE 8:e65835

8. Martineau AR, Jolliffe DA, Hooper RL et al (2017) Vitamin D supplementation to prevent acute respiratory tract infections: systematic review and meta-analysis of individual participant data. BMJ 356:i6583

9. Pérez Castrillón JL, Casado E, Corral Gudino L et al (2020) COVID-19 and vitamin D. Position paper of the Spanish Society for Bone Research and Mineral Metabolism (SEIOMM). Rev Osteoporos Metab Miner 12:155-159

10. Kaufman HW, Niles JK, Kroll MH, Bi C, Holick MF (2020) SARS-CoV-2 positivity rates associated with circulating 25-hydroxyvitamin D levels. PLoS ONE 15:e0239252. https:// doi.org/10.1371/journal.pone.0239252

11. Meltzer DO, Best TJ, Zhang H, Vokes T, Arora V, Solway J (2020) Association of vitamin D status and other clinical characteristics with COVID-19 test results. JAMA Netw Open 3(9):e2019722. https://doi.org/10.1001/jamanetworkopen.2020.19722

12. Merzon E, Tworowski D, Gorohovski A, Vinker S, Golan Cohen A et al (2020) Low plasma 25(OH) vitamin D level is associated with increased risk of COVID-19 infection: an Israeli populationbased study. FEBS J. https://doi.org/10.1111/febs.15495

13. Radujkovic A, Hippchen T, Tiwari-Heckler S, Dreher S, Boxberger M, Merle U (2020) Vitamin D deficiency and outcome of COVID-19 patients. Nutrients 12:2757. https://doi.org/10.3390/ nu12092757

14. Abrishami A, Dalili N, Torbati PM, Asgari R, Arab-Ahmadi M, Behnam B et al (2020) Possible association of vitamin D status with lung involvement and outcome in patients with COVID-19: a retrospective study. Eur J Nutr 30:1-9. https://doi.org/10.1007/ s00394-020-02411-0

15. Luo X, Liao Q, Shen Y, Li H, Cheng L (2021) Vitamin D deficiency is associated with COVID-19 incidence and disease severity in Chinese people. J Nutr 151:98-103

16. Bassatne A, Basbous M, Chakhtoura M, El Zein O, Rahme M, El-Hajj FG (2021) The link between COVID-19 and vitamin D (VIVID): a systematic review and meta-analysis. Metab Clin Exp 119:154753

17. Kazemi A, Mohammadi V, Aghababaee SK, Golzarand M, Clark CCT, Babajafari S (2021) Association of vitamin D status with SARS-CoV2 infection or COVID-19 severity: a systematic review and meta-analysis. Adv Nutr. https://doi.org/10.1093/advances/ nmab012

18. Pereira M, Dantas Damascena A, Galvão Azevedo LM, de Almeida OT, da Mota SJ (2020) Vitamin D deficiency aggravates COVID-19: systematic review and meta-analysis. Crit Rev Food Sci Nutr. https://doi.org/10.1080/10408398.2020.1841090

19. Hernández JL, Nan D, Fernández-Ayala M, García-Unzueta M, Hernández-Hernández MA, López-Hoyos M et al (2020) Vitamin D status in hospitalized patients with SARS-CoV2 infection. J Clin Endocrinol Metab 20:1-11. https://doi.org/10.1210/clinem/ dgaa733

20. Annweiler G, Corvaisier M, Gautier J et al (2020) Vitamin D supplementation associated to better survival in hospitalized frail elderly COVID-19 patients: the GERIA-COVID quasi-experimental study. Nutrients 12:3377

21. Ling SF, Broad E, Murphy R, Pappachan JM, Pardesi-Newton S, Kong MF et al (2020) High-dose cholecalciferol booster therapy is associated with a reduced risk of mortality in patients with COVID-19: a cross-sectional multi-centre observational study. Nutrients 12:3799. https://doi.org/10.3390/nu12123799
22. Cereda E, Bogliolo L, Lobascio F et al (2019) Vitamin D supplementation and outcomes in coronavirus disease 2019 (COVID19) patients from the outbreak area of Lombardy, Italy. Nutrition 2020:111055. https://doi.org/10.1016/j.nut.2020.111055

23. Entrenas Castillo M, Entrenas Costa LM, Vaquero Barrios JM et al (2020) Effect of calcifediol treatment and best available tehrapy on intensive care unit admission and mortality among patients hospitalized for COVID-19: a pilot randomized clinical study. J Steroid Biochem Mol Biol 203:105751

24. Rastogi A, Bhansali A, Khare N et al (2020) Short term, highdose vitamin D supplementation for COVID-19 disease: a randomised, placebo-controlled, study (SHADE study). Postgrad Med J. https://doi.org/10.1136/postgradmedj-2020-139065

25. Murai IH, Fernandes AL, Sales LP et al (2021) Effect of a single high dose of vitamin D3 on hospital length of stay in patients with moderate to severe COVID-19. A randomized clinical trial. JAMA. https://doi.org/10.1001/jama.2020.26848

26. Oristrell J, Oliva JC, Subirana I, Casado E, Domínguez D, Toloba A et al (2021) Association of calcitriol supplementation with reduced COVID-19 mortality in patients with chronic kidney disease:a population-based study. Biomedicines 9:509. https:// doi.org/10.3390/biomedicines9050509

27. Williamson EJ, Walker AJ, Bhaskaran K et al (2020) Factors associated with COVID-19-related death using OpenSAFELY. Nature 584:430-443

28. Sekhon JS (2011) Multivariate and propensity score matching software with automated balance Optimization: the matching package for R. J Stat Soft 42:1-52

29. Levey AS, Stevens LA, Schmid CH et al (2009) CKD-EPI (Chronic Kidney Disease Epidemiology Collaboration). A new equation to estimate glomerular filtration rate. Ann Intern Med 150:604-612

30. Yoshida K, Bohn J. Package 'tableone' for R. Accessible online at https://cran.r-project.org/web/packages/tableone/tableone.pdf

31. Therneau T (2020) A package for survival analysis in R. R package version 3.2-7, https://CRAN.R-project.org/package=survival

32. Kassambara A, Kosinski M, Biecek $P$ et al. Survminer: drawing survival curves using 'ggplot2', https://CRAN.R-project.org/packa ge $=$ survminer

33. Dusso AS, Tokumoto M (2011) Defective renal maintenance of the vitamin D endocrine system impairs vitamin D renoprotection: a downward spiral in kidney disease. Kidney Int 79:715-729

34. Greiller CL, Martineau AR (2015) Modulation of the immune response to respiratory viruses by vitamin D. Nutrients 7:4240 4270. https://doi.org/10.3390/nu7064240

35. Kong J, Zhu X, Shi Y et al (2013) VDR attenuates acute lung injury by blocking Ang-2-Tie-2 pathway and renin-angiotensin system. Mol Endocrinol 27:2116-2125. https://doi.org/10.1210/ me.2013-1146

36. Dancer RCA, Parekh D, Lax S et al (2015) Vitamin D deficiency contributes directly to the acute respiratory distress syndrome (ARDS). Thorax 70:617-624. https://doi.org/10.1136/thora xjnl-2014-206680

37. Xu J, Yang J, Chen J et al (2017) Vitamin D alleviates lipopolysaccharide-induced acute lung injury via regulation of the reninangiotensin system. Mol Med Rep 16:7432-7438. https://doi.org/ 10.3892/mmr.2017.7546

38. Loucera C, Peña-Chilet M, Esteban-Medina M, Muñoyerro-Muñiz D, Villegas R, López-Miranda J et al (2021) Real-world evidence of calcifediol use and mortality rate of COVID-19 hospitalized in a large cohort of 16,401 Andalusian patients. MedRxiv Preprint. https://doi.org/10.1101/2021.04.27.21255937

39. Haussler MR, Professor R, Jurutka PW et al (2011) Vitamin D receptor (VDR)-mediated actions of $1 \alpha, 25(\mathrm{OH})_{2}$ vitamin $\mathrm{D}_{3}$ : genomic and non-genomic mechanisms. Best Pract Res Clin Endocrinol Metab 25:543-559 
40. Brunette MG, Chan M, Ferriere C, Roberts KD (1978) Site of $1,25(\mathrm{OH}) 2$ vitamin D3 synthesis in the kidney. Nature 276:287-289

41. Lely AT, Hamming I, van Goor H, Navis GJ (2004) Renal ACE2 expression in human kidney disease. J Pathol 204:587-593

42. Werion A, Belkhir L, Perrot M, Schmit G, Aydin S, Chen Z et al (2020) SARS-CoV-2 causes a specific dysfunction of the kidney proximal tubule. Kidney Int 98:1296-1307

43. Armaly Z, Kinaneh S, Skorecki K (2021) Renal manifestations of COVID-19: physiology and pathophysiology. J Clin Med 10:1216. https://doi.org/10.3390/jcm10061216
Publisher's Note Springer Nature remains neutral with regard to jurisdictional claims in published maps and institutional affiliations. 